\section{Advancing Science while Training Undergraduates: Recommendations from a Collaborative Biology Research Network}

\author{
Danielle Jensen-Ryan, ${ }^{\dagger}$ Courtney J. Murren, ${ }^{\ddagger}$ Matthew T. Rutter, ${ }^{\ddagger}$ and \\ Jennifer Jo Thompson ${ }^{\S *}$ \\ ${ }^{\dagger}$ Department of Math and Sciences, Laramie County Community College, Cheyenne, WY 82007; \\ 'Department of Biology, College of Charleston, Charleston, SC 29424; and \$Department of Crop \\ and Soil Sciences, University of Georgia, Athens, GA 30602
}

\begin{abstract}
Biology research is becoming increasingly dependent on large-scale, "big data," networked research initiatives. At the same time, there has been a corresponding effort to expand undergraduate participation in research to benefit student learning and persistence in science. This essay examines the confluence of this trend through eight years of a collaboration within a successful biology research network that explicitly incorporates undergraduates into large-scale scientific research. We draw upon interviews with faculty in this network to consider the interplay of scientific and pedagogical objectives at the heart of this undergraduate-focused network research project. We identify ways that this network has expanded and diversified access to scientific knowledge production for faculty and students and examine a goal conflict that emerged around the dual objectives of mentoring emerging scientists while producing high-quality scientific data for the larger biology community. Based on lessons learned within this network, we provide three recommendations that can support institutions and faculty engaging in networked research projects with undergraduates: (1) establish rigorous protocols to ensure data and database quality, (2) protect personnel time to coordinate network and scientific processes, and (3) select appropriate partners and establish explicit expectations for specific collaborations.
\end{abstract}

\section{INTRODUCTION}

Humanity faces "grand challenges" of climate change, (re-)emerging disease, and resource scarcity, among others (Barnosky et al., 2016). Given the magnitude of these challenges, scientific knowledge creation is transitioning to a new era-from one steeped in disciplinary silos to transdisciplinary networked science (Nielsen, 2011). Concurrent with the rise in networked research, science education research has increasingly recognized the value of undergraduate research participation for student learning and persistence in science (Laursen et al., 2010; American Association for the Advancement of Science [AAAS], 2011). Situated at the confluence of these trends, this essay contributes to understanding how biology educators can effectively include undergraduate research participants in collaborative, cross-institutional, networked research projects. Drawing upon 8 years of a collaboration within a successful biology research network (BRN) that explicitly incorporates undergraduates into large-scale scientific knowledge production, we consider how the inclusion of undergraduates contributes to the overall scientific and pedagogical objectives of networked research. We identify synergies and challenges at the interface of undergraduate research participation and scientific knowledge production, and offer pragmatic recommendations to support the growth of additional effective undergraduate-focused research networks going forward.
Graham F. Hatfull, Monitoring Editor Submitted May 14, 2020; Revised Sep 21, 2020; Accepted Sep 29, 2020

CBE Life Sci Educ December 1, 2020 19:es13 DOI:10.1187/cbe.20-05-0090

*Address correspondence to: Jennifer Jo Thompson (jjthomp@uga.edu).

(c) 2020 D. Jensen-Ryan et al. CBE-Life Sciences Education $\odot 2020$ The American Society for Cell Biology. This article is distributed by The American Society for Cell Biology under license from the author(s). It is available to the public under an Attribution-Noncommercial-Share Alike 3.0 Unported Creative Commons License (http://creativecommons.org/licenses/ by-nc-sa/3.0).

"ASCB $® "$ and "The American Society for Cell Biology $\AA^{\circledR}$ are registered trademarks of The American Society for Cell Biology. 


\section{The Emergence of Networked Research in Biology}

Networked science resembles a team progressing toward common research goals; it is commonly structured to address complex socioscientific questions that can only be answered through the production and analysis of massive data sets (Rainie and Wellman, 2012; Mo et al., 2015; Simmons et al., 2016). Networked research groups are also often multidisciplinary-even transdisciplinary (Jahn et al., 2012)—spanning institutions, stakeholders, and geographic sites (Mo et al., 2015). Further, because communication within these networks is largely virtual (video conferencing, online workbooks, etc.; Rainie and Wellman, 2012), networked research offers opportunities to foster the emergence of cyber-infrastructure and e-science (Hey and Trefethen, 2008), reduce anthropogenic carbon emissions associated with professional research travel (Waring et al., 2014), and expand participation for researchers with fewer resources for travel and/or other travel constraints.

Within biology, the shift from "little science to Big Science" (de Solla Price, 1963) gained traction in the 1990s with the mapping of the Caenorhabditis elegans genome (Ankeny, 2001); the rise of model organism communities (Ankeny and Leonelli, 2012); and advances in genome annotation (Stein, 2001). The Human Genome Project further transformed the parameters of multi-institutional scientific production (Bowker and Star, 1999). These, and other networked research projects, opened new avenues for rapidly sharing biological research data, often well before journal publication, a feat valued as both technologically feasible and, after some contention, professionally acceptable (Jones et al., 2018). Building on these projects, biologists continue to collaborate across institutions as certain research questions (and professional standards) become increasingly dependent on large-scale networked initiatives (Nielsen, 2011).

\section{Previous Studies of Networked Research}

Studies of networked research have examined large-scale data production, patents, coauthorship, citation, and grant productivity (Cummings and Kiesler, 2005). Organizational researchers have led this field, documenting the characteristics of "Big Science" (Bos et al., 2008)—including how people organize across time and space (Hinds and Kiesler, 2002), the role of trust (Jarvenpaa and Leidner, 1999) and communication (De Sanctis and Mong, 1998), the coordination and management of collaborations (Cummings and Kiesler, 2005), and how institutional resources shape the nature of the collaboration (BinzScharf et al., 2014).

Sociologists and anthropologists, including the pioneers of science and technology studies, have examined networked research with a focus on the foundations of scientific knowledge production, especially in research labs (Latour and Woolgar, 1987; Binz-Scharf et al., 2014). These studies examine the micro- and macrolevel interactions to understand collaborative dynamics present in research networks-finding, for example, that network hierarchies ultimately impact access to critical academic opportunities, such as invited presentations and publication in high-impact journals (Alačc, 2011; Binz-Scharf et al., 2014).

Within biology, ethnographic scholarship has examined how model organism-based knowledge has scaled to broader medical sciences, creating generalizable and actionable results (Ankeny, 2000; Nelson, 2017). Other networked research studies have examined how practices, materiality, politics, theories, moral economies, and cultures-including norms of rapid and prepublication data sharing - have evolved (cf. Jones et al., 2018). For example, Leonelli's (2011) work described how information circulates in biology, with a focus on the creation and use of databases for collaboration. Sarma et al. (2018) recount the history of Caenorhabditis elegans, with a focus on evolving database systems like OpenWorm, while Jones et al. (2018) review the Human Genome Project and corresponding Bermuda Principles, to name a few.

\section{Challenges of Networked Research}

Despite the benefits of diverse expertise and research momentum of large groups, scholars participating in large-scale networked research projects face unique challenges when collaborating across disciplines and geographic distance (Bos et al., 2008). Scholars from different disciplines may lack a shared foundation around key issues, literature, methodologies, and research practices_creating opportunities both for miscommunication and growth in scientific settings and demanding extended periods to forge common understandings and a shared language (Cummings and Kiesler, 2005). Other studies identify communication issues that emerge (Bos et al., 2001; Olson and Olson, 2003) and networked research groups' preferences for face-to-face meetings over digitally mediated communication (Bos et al., 2008). Challenges also stem from the institutional structures that house networked research. Mo et al. (2015) found coordinating research activities across individuals employed in large bureaucracies, such as universities or governmental organizations, difficult and slow. Further, traditional academic rewards, tied to tenure and promotion, implicitly discourage cross-institutional collaboration (Nielsen, 2011; BinzScharf et al., 2014). Despite these challenges, network research is transforming collaboration and scientific knowledge production in valuable and important ways, as we described earlier.

\section{Undergraduate Involvement in Networked Biology Research}

The Vision and Change report on undergraduate education in biology (AAAS, 2011) called for improving biology instruction by integrating undergraduate research into curricula. This report cited many advantages for undergraduates, including lasting learning, the ability to evaluate scientific claims in everyday life, confidence and motivation in learning, and bolstering personal identification as a scientist and interest in scientific careers (cf. Laursen et al., 2010). Overall, participating in an authentic research experience-whether in the context of undergraduate research experiences (UREs) or course-based undergraduate research experiences (CUREs)-positively impacts undergraduates' scientific interest and self-confidence in performing lab tasks as compared with students experiencing a traditional biology "cookbook" lab (Brownell et al., 2012).

Until recently, undergraduates were seldom incorporated into high-level biology networked research groups that focus on addressing large-scale questions with "big data" and produce coauthored peer-reviewed publications (Hara et al., 2003; Simmons et al., 2016). Over the last decade, however, networked research groups that explicitly include undergraduates have emerged, yielding new opportunities for higher education and science, technology, engineering, and mathematics (STEM) 
learning (Hanauer and Dolan, 2014; Rutter et al., 2019). For example, the Howard Hughes Medical Institute-funded Science Education Alliance-Phage Hunters Advancing Genomics and Evolutionary Science (SEA-PHAGES) program incorporates early-career undergraduate students to aid them in understanding viral diversity and evolution (Hanauer et al., 2017). More than 100 institutions participate in SEA-PHAGES, with genome findings reported in more than 70 peer-reviewed publications, many with student coauthors (Hanauer et al., 2017). Including undergraduates can both facilitate student learning about research design and analysis and free faculty to dedicate time to other research activities - a two-for-one advantage of merging experiential learning with innovative and productive research (Bowne et al., 2011). As BRNs increasingly include undergraduates, students have the opportunity to contribute to scientific knowledge production, interact and share insights with students and faculty at other institutions, and develop scientific identity and cultural capital (Bowne et al., 2011; Thompson et al., 2016; Thompson and Jensen-Ryan, 2018). Undergraduate BRNs also effectively prepare student for scientific careers (Simmons et al., 2016).

\section{Learning from an Undergraduate-Focused BRN: A Case Study}

This essay emerges from a longitudinal research collaboration within a multi-institutional BRN (2011-present). The BRN began as an interdisciplinary collaboration among five faculty at three primarily undergraduate institutions. As of 2019, the research network had expanded to 17 faculty supervising undergraduate research at 15 institutions-including research-intensive, primarily undergraduate, 2-year community college, and minority-serving institutions in the United States and Canada (Table 1) with project management located at one of the original institutions. As of 2019, 123 undergraduates across 15 institutions had also participated in UREs in this network, working for credit and pay.

The BRN has ambitious scientific and pedagogical objectives: to build a robust phenotypic data set using a distributed science model while training undergraduates as researchers (Rutter et al., 2019). From the beginning, undergraduates have been central to the production of data and scientific knowledge. From the outset, the BRN has focused on a long-term laboratory research model, spanning 1 to 4 years. In general, undergraduates join the project as sophomores or juniors and are recruited through peers, STEM-focused student programs, or courses with BRN faculty (including project-related CUREs, which are outside the scope of the current paper). Upon joining the BRN,

TABLE 1. Biology research network faculty, institution, and research information

\begin{tabular}{ll}
\hline Faculty demographics $(\boldsymbol{n}=\mathbf{1 7})$ & No. (\%) \\
\hline Gender & \\
$\quad$ Female & $9(53 \%)$ \\
$\quad$ Male & $8(47 \%)$ \\
Institution type $(n=15)$ & \\
$\quad$ Research-intensive university & $2(13 \%)$ \\
Primarily undergraduate institution & $9(60 \%)$ \\
Historically Black college/university & $1(7 \%)$ \\
Two-year institution or community college & $3(20 \%)$ \\
\hline
\end{tabular}

undergraduates learn standardized project protocols in face-toface laboratory settings, while also learning how their work contributes to the collective project goals. At the network level, a science manager based at the lead institution has provided in-person or virtual training to faculty and undergraduate researchers, who continue to teach the protocols to future cohorts of undergraduate researchers. Virtual protocol "refresher" training is common across the network. The project's rigorous protocols standardize experimental design, data collection, and data quality before data are entered into in a shared database (Murren et al., 2019; Rutter et al., 2019). Over time, students may develop independent projects that build upon their experience with a team project, interest in developing an independent research question, and research requirements at each institution. At most of the BRN institutions, faculty directly oversee undergraduate research.

We draw upon this BRN as an example of a successful network, with effective communication and a general sense of trust among faculty who have engaged in iterative problem solving over the course of the project. There were no models of an undergraduate-focused research network studying evolutionary ecology in plants before the establishment of this BRN. Over the years, it has expanded and diversified access to scientific knowledge production-for faculty and students from large public institutions, public and private primarily undergraduate institutions, as well as 2-year, minority-serving, and historically black institutions. Securing funding for this research, expanding publicly available data for the plant science community, training hundreds of undergraduates in research, and developing CUREs and a summer URE are all evidence of the network's success.

\section{Examining the Impact of Undergraduate Participation in a Biology Research Network}

Over the course of the collaboration, the social science team (D.J.-R. and J.J.T.) conducted participant observation and several waves of interviews with students and faculty within the network. Between 2012 and 2018, our team conducted a total of 23 interviews with 13 faculty (93\% of the total faculty involved in the BRN at the time), which represents a "complete target population" purposive sampling strategy (Patton, 2015). Here, we draw heavily on the interviews with five "core faculty" who have been involved in the research since it began and are thus able to provide a longitudinal perspective on the network; triangulating this information with other faculty interviews provides unique insight into the development of an undergraduate-focused research network and its development over the course of several years (Flick, 2004).

Core faculty were interviewed three times over 5 years, in 2012, 2016, and 2018. This essay draws from the full data setbut particularly from interviews conducted with core faculty in 2018. The objective of the 2018 core faculty interviews was twofold: 1) to understand how the scientific and pedagogical goals of the BRN evolved over the course of the project and 2) to examine how the explicit inclusion of undergraduates impacted the research project (see Faculty Interview Guide in Supplemental Material). Interviews lasted 60-120 minutes. After each interview, the interviewer made detailed notes, including the context and content of the interview, and connections to issues in the literature on networked research. All interviews were audio-recorded and transcribed verbatim. 
Our analysis began with a review of the interview transcripts for accuracy before they were imported into Atlas.ti (qualitative data management software, Scientific Software Development $\mathrm{GmbH}$ ). In Atlas.ti, we systematically read and indexed each interview-using versus coding and concept coding approaches (Saldaña, 2016; see code list in Supplemental Materials). Versus coding, which focuses on understanding competing goals, or opposing norms/values in a situation, allowed us to identify themes related to network synergies and challenges. Concept coding, which focuses on identifying the ideas behind specific instances in the data, allowed us to further investigate the relationship between scientific and pedagogical goals of the project. Interviews were initially coded by D.J.-R. and cross-checked for accuracy by J.J.T. Regular meetings occurred to discuss areas of ambiguity that we flagged during indexing and resolve any discrepancies among identified themes. Our code list and definitions stabilized after two faculty interviews were fully indexed. During this process, we added codes, refined code definitions, collapsed code categories, and re-indexed interviews to reflect the data and to ensure consistency in our analysis (Saldaña, 2016).

We then triangulated our thematic results. First, we compared the full data set (all faculty interviews) with the 2018 faculty interviews to contextualize our analysis (Flick, 2004). The longitudinal nature of this collaboration offers the ability to investigate the emergence and development of themes across interviews with faculty and students over the course of 8 years. Though earlier faculty interviews were indexed for different purposes, including student outcomes in the BRN (see Thompson et al., 2016; Thompson and Jensen-Ryan, 2018), we were able to interrogate and corroborate our analysis by rereading all former faculty interviews with our current analysis in mind. Second, after developing initial interpretations of the data, J.J.T. and D.J.-R. discussed and refined the findings in consultation with M.T.R. and C.J.M., who train and mentor students while also coordinating the BRN, to deepen our analysis and develop recommendations within the context of the project as a whole (Creswell and Miller, 2000). Including two core faculty in the collaborative process of this paper allowed for robust respondent validation of our interpretations and conclusions, an approach that Maxwell describes as "the single most important way of ruling out the possibility of misinterpreting the meaning of what participants say and do" (2013, p. 126). This study was approved by the University of Georgia's Institutional Review Board, and all participants provided informed consent.

\section{Understanding the Effect of Undergraduate Participation in Networked Research}

Based upon our analysis of the BRN, we identified two primary themes associated with undergraduate research participation in networked research producing "Big Science": synergies and goal conflicts at the student-science interface. We present each theme and its subthemes separately, with specific quotations from the research interviews, and follow with a set of recommendations for biology educators involved in research networks with undergraduates.

Theme One: Synergies at the Student-Science Interface. We identify three categories of positive interactions between students and science that enhance the value of the work accom- plished within the BRN. Simply put, we find that 1) students enhance the science; 2) participation in science enhances students; and 3) professional development for faculty supports their success as both scientists and mentors.

In interviews across the data set, faculty characterized the inclusion of undergraduate researchers as vital to building a robust scientific database and addressing large-scale research questions. Faculty described the ways undergraduates substantively contributed to the project by helping to refine and implement scientific protocols while also training peers to conduct research. For example, one core faculty member emphasized the "quantum change" in the research network protocols based upon a student's novel innovation, stating: "What there has been ... is improving efficiency ... [this student] really changed that dramatically. Quantum change ... I used to have to have three students at the [lab] bench to get stuff done, but she made it so one student could do it." This student's contribution to the network ultimately saved other researchers "countless hours" of work. Another faculty member described one student as a "right-hand person" and particularly skilled at "seeing unusual branching, unusual traits" in plants during data collection. Several faculty also described how students positively impacted the "culture" of the lab and even the trajectory of research, while also being exposed to personal and professional development opportunities_-including traveling out of state for the first time; building relationships that facilitate strong faculty recommendations; and communicating their work through posters, presentations, and publications. These are tangible contributions made and benefits gained by undergraduates in the BRN.

Elsewhere, we have documented that undergraduate researchers within this network developed multiple forms of capital through their participation in scientific researchincluding technical and analytical skills, the ability to think and work like a scientist, and multidimensional relationships with mentors and peers (Thompson et al., 2016). Further, while undergraduates join their research labs with varying levels of cultural capital in science (e.g., exposure and experience), we found that faculty in this network largely offer students the kind of support and recognition needed to help them expand their capital and strengthen their identities as scientists (Thompson and Jensen-Ryan, 2018).

Beyond the direct synergies of students contributing to the scientific goals of the project and students' research participation contributing to their development as scientists, we also identified synergies that support faculty in their mentorship of students as a key facet of their success as scientists. Project leadership has expanded teaching, research, and professional development opportunities for faculty by hosting project meetings, developing protocols and materials for implementing project-related CURES, supporting experimental designs that fit faculty infrastructure constraints while meeting project goals, and mentoring faculty with high teaching contact-hour positions in maintaining teaching and research obligations. As a result, this project has supported faculty in securing tenure-track job offers, as well as promotion and tenure; training hundreds of students through lab- and course-based research, ultimately yielding dozens of national and international posters and oral presentations, and peer-reviewed papers (many with undergraduates as copresenters and/or authors); and producing a publicly 
available database with more than 500,000 phenotypes for use by the scientific community.

Theme Two: Goal Conflicts at the Student-Science Interface. Despite the evident successes of this network, it is valuable to examine the challenges networks may face in order to enhance the success of networked research more broadly. This attention is not intended to minimize the specific accomplishments of this network or to discourage networked research groups from incorporating undergraduates in large-scale research efforts. To the contrary, our analysis aims to identify recommendations based on lessons learned within this network to support undergraduate-focused networked research groups in achieving both their scientific and pedagogical objectives and to encourage funding agencies and institutions to support these networks with the resources needed to maximize their success on both fronts.

We frame the central challenge identified in our data as a goal conflict, that is, two or more goals representing "divergent preferences" (Cosier and Rose, 1977, p. 378) and often "compet[ing] for the same resources such as time or money" (Kelly et al., 2015, p. 213). Notably, research on goal conflicts suggests that "conflicting goals [do] not appear to be inherently less attainable than non-conflicting goals" (Boudreaux and Ozer 2013, p. 440). Nevertheless, pursuing competing goals can be stressful and thus demand strategies for navigating (and perhaps integrating) areas of potential conflict and competition.

From the beginning, BRN faculty shared a dual commitment to undergraduates' skill development while contributing to larger, scientific objectives. However, in their reflective interviews, core faculty characterized this dual commitment to scientific productivity and student development as a central goal conflict they had to navigate. This goal conflict was amplified by the ways that institutions differentially structure faculty priorities toward the scientific and pedagogical goals of the project. Although both goals are worthwhile, at times they compete for limited resourceincluding faculty time and attention. Two subthemes with quotes from the "core faculty" are provided in the following sections to demonstrate the goal conflict that emerged in our research.

\section{GOAL CONFLICT: SCIENTIFIC PRODUCTIVITY}

Core faculty discussed the challenge they faced in focusing on larger scientific questions, and research responsibilities like data analysis, database management, and producing publications and presentations due to the time spent training and mentoring undergraduates. This has been documented elsewhere as a particular challenge for faculty at undergraduate-focused institutions with high teaching loads and few or no graduate students or postdocs to assist with research, writing, and mentoring undergraduates (Binz-Scharf et al., 2014; Nielsen, 2011). While acknowledging the value of students' experiences one faculty member described a cost to scientific productivity,

What we [faculty] have spent our time doing is more of training the students ... I think that many of us ... started thinking more about the machine and its moving parts, and so much of it are ...the experiences the students were having, the interactions among students ..., we focused on oiling and maintaining our machine instead of using the machine to answer the questions.

\section{Another agreed,}

What we learned going in is that we were a little over-ambitious with the volume of what we could accomplish ... if you're going to have the bulk of the actual work done by actual undergraduates, it's going to be a lot slower than you would envision ... things haven't moved to the pace we would like.

Although the feeling that "things haven't moved to the pace we would like" is common across research, here faculty experienced it as a goal conflict between undergraduate development and overall scientific productivity - with several communicating the challenges of simultaneously presenting scientific work and publishing articles while training and mentoring a high volume of undergraduate researchers.

\section{GOAL CONFLICT: STUDENT DEVELOPMENT}

Every research project faces unexpected problems, and even well-trained researchers make mistakes; yet several core faculty in this network specifically emphasized the unique challenges of simultaneously training and mentoring novice scientists while also producing high-quality data for the broader scientific community. One core faculty member focused on this point, saying, "It's been more of a teaching thing, and less of a getting the work done ... That's just having students."

Recognizing the value of the pedagogical aims, one faculty member embraced a shift in focus away from scientific productivity and toward student development, stating:

It became frankly necessary to expand what we were doing scientifically for it to be useful for our students ... once I got the students going, I'm like wow, we need to have something intellectual here going on. And so our science evolved into finding new ways to get the students to ask their own questions ... So when you start from where we were to where we are now, it is quite the shift in terms of what our scientific focus has been.

This faculty member went on to say:

My contribution is pedagogical, is coming up with the ways to integrate these things into classes ... Our original intent was to contribute hundreds and hundreds of data points ... [Now] that's frankly not as important for us.

In the end, experiencing trade-offs and challenges in the context of collaborative research is not unusual (Bos et al., 2001; Olson and Olson, 2003), yet it highlights the complexity of the dual goals at the heart of a research collaboration that is simultaneously training large numbers of undergraduate researchers and advancing significant scientific research objectives.

\section{Recommendations for Maximizing Success in Undergraduate-Focused Research Networks}

In interviews, faculty reflected on the lessons learned within eight years of collaboration and offered several applied recommendations that could further enhance networks like thisespecially with regard to navigating the dual goals of undergraduate participation and scientific productivity. Drawing on our longitudinal research, our recommendations are intended 


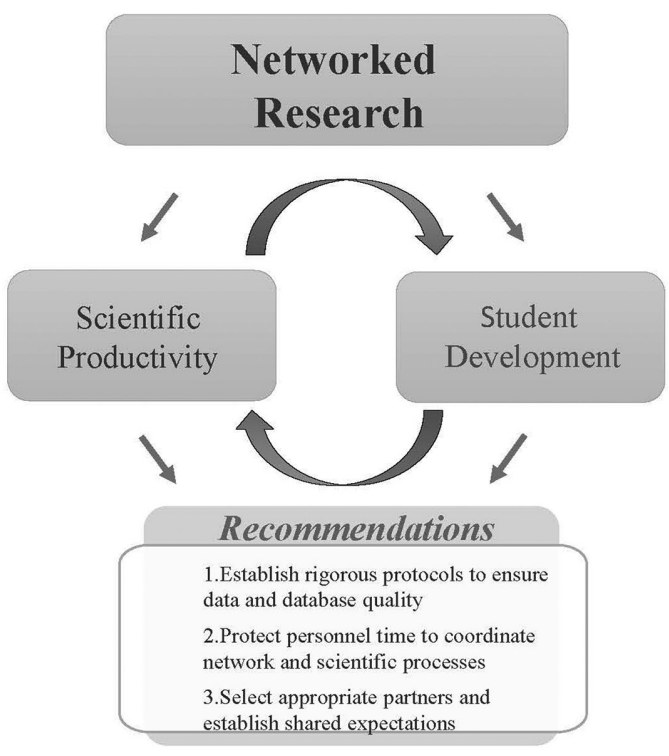

FIGURE 1. Recommendations for undergraduate-focused research networks.

to aid those engaged in networked research with undergraduates and those planning large-scale research projects in securing support and structuring collaborations to maximize success for both students and science (Figure 1).

Recommendation One: Establish Rigorous Research Protocols to Ensure Data and Database Quality. As in any research project, the faculty on this project have always been concerned about ensuring the quality of the research and resulting database. Through early-phase experimentation, core faculty developed standardized protocols to structure experiments and data collection (see Rutter et al., 2019). These include measuring common traits and using multiple common controls to account for variation from site to site-something vital for distributed research. Notably, undergraduate researchers played essential roles in protocol development during this phase of research and in mentoring new students throughout. To ensure database quality, the network also developed protocols for data replication, independent proofreading, and flagging unusual values for further review before data are entered into the shared database.

Although there is evidence that some science students prefer structured projects likely to lead to publication (Alkaher and Dolan, 2014), increased structure can reinforce "cookbook" approaches to data production, reducing the potential for student ownership and experimentation in the context of learning (Brownell et al., 2012). Authentic scientific experiences retain students in STEM and enhance their likelihood of success, especially among underrepresented students (Russell et al., 2007; Bangera and Brownell, 2014; Carpi et al., 2017). Within the $\mathrm{BRN}$, increased standardization of protocols supported the development of project-related CUREs as an efficient and effective way to engage a large number of students in research simultaneously. CUREs have also supported project efforts to build in the replication and review of data before they are entered into the database-engaging students in the process of ensuring data quality. Though the BRN protocols are stringent and structured to ensure data quality, they also provide the necessary foundation for undergraduates to design independent research projects that build on interesting results of collective experimentation. Thus, this research network safeguards scientific credibility while simultaneously encouraging authentic student discovery, design, and innovation.

Recommendation Two: Protect Personnel Time/Effort to Coordinate Network and Scientific Processes and Outputs. As any research network grows beyond a small number of close collaborators, there is increased need for structure to support the network itself. Although this network established rigorous scientific protocols to safeguard data and database quality, as well as structures for undergraduate training, core faculty indicated that they did not anticipate how difficult and time-consuming network management itself would be. Networked research requires a great deal of administration and coordination to maintain collaborations and ensure scientific productivity. In the case of undergraduate-focused networks, it also requires ongoing attention to student development and mentorship. Faculty at the center of the network face unique obligations to maintain grant and institutional funding for staff positions and the research itself, along with reporting to funding agencies and maintaining a public face for the work. With this in mind, we encourage networks to seek (and institutions to approve) course releases for faculty who oversee network management and coordination.

In addition, we strongly recommend developing a structure to delegate network responsibilities, guide management decisions, and protect the time of personnel (especially faculty) fulfilling these responsibilities. We describe here several network roles that core faculty described as important for the optimization of work in this project and others like it.

Science Manager. Several years into the project, as new institutions were coming on board, a full-time manager was hired in the BRN to further expand tools to standardize study protocols, execute training across institutions, order and distribute research materials, coordinate meetings between faculty, and finally, collate and standardize data. Overall, core faculty credit the science manager with drastically improving the "lag" in network productivity by helping to organize and manage the "mountain of data." Because faculty have substantial contact-hour and on-campus service obligations, it was critical that this staff member have broad availability during the week to manage science-related issues.

Data Manager. This project was fortunate to have a faculty member with relevant expertise create and manage the online database. Given the need to collate, standardize, and manage large, often public-facing databases common in networked research projects, having experienced personnel focus solely on data standardization, programming, and database management would reduce data bottlenecks and enable faculty to focus on scientific productivity and student development, rather than the technical network itself.

Network Manager. Finally, our results indicate that maintaining cross-institutional network administration and communication (e.g., completing administrative reporting, coordinating meetings and conference calls, and facilitating information flow) 
can be time-consuming and logistically difficult. Dedicating personnel to enhancing cross-institutional relationships among network participants, including students, would provide the connectivity that often falls to already-overcommitted faculty.

Depending on the network and institutions involved, these roles could be fulfilled by faculty, staff, graduate students, and/ or postdoctoral researchers (Delamont and Atkinson, 2001; Dolan and Johnson, 2010). Certainly, hiring additional personnel to fulfill these roles would enable faculty to focus on mentoring students while maintaining scientific productivity. Although additional personnel would have significantly aided this network as it hit bottlenecks (especially before the science manager was hired), it is important to emphasize that this network has been successful with minimal additional staff. Although institutions and funding agencies may be reluctant to provide durable funding for staff or significant course releases for faculty, there is a vital need to support dedicated time from network personnel for their coordinating efforts, and we strongly encourage institutions and funders to recognize and respond to this urgent need.

Recommendation Three: Select Appropriate Partners and Establish Shared Expectations. Several core faculty discussed the need for network partners to have "shared motivation" and shared research goals. This network drew partners from past relationships, geographic proximity, and direct requests for inclusion. Though this approach expanded the network considerably, faculty sometimes diverged in how they prioritized student learning versus scientific productivity. Recognizing this, several core faculty recommended developing a process to assess fit for partners in particular network roles. When developed deliberately, this process helps articulate network priorities and diverse partnerships, thus supporting a network's longterm objectives. Importantly, any selection process must not oversimplify and inadvertently exclude potential collaborators due to implicit bias or a lack of research infrastructure (Greenwald and Banaji, 1995). In this BRN, faculty at minority-serving and teaching-focused institutions have been valuable collaborators. We also recommend low-stakes "trial periods" during which either party could walk away from network partnership.

Unexpected changes in goals may emerge over time with existing or new partners. For example, partners may have substantial changes in their own institutional obligations (e.g., taking on administrative roles), come to realize they do not share norms around undergraduate participation (Cummings and Kiesler, 2005), or consistently experience research challenges leading to missed milestones (Sonnenwald, 2008). While no panacea, establishing a memorandum of understanding with clearly articulated expectations can better ensure shared expectations among partners and enhance communication (Sonnenwald, 2008). Letters of commitment from partner institutions can also help mitigate concerns from faculty who may find their research collaborations unsupported by their home institutions. Certainly, network and scientific visions may shift over time; as such, it is vital to build in an ongoing process for communication and amendment.

\section{CONCLUSION}

Biology research is becoming increasingly dependent on largescale, big data, networked research initiatives that are expand- ing undergraduate research in response to calls such as Vision and Change (AAAS, 2011). In this essay, we draw upon interviews conducted during longitudinal research collaboration within a BRN to examine the interplay of scientific and pedagogical objectives at the heart of this undergraduate-focused network research project. The project has explicit objectives to both expand undergraduate research participation and produce high-quality scientific data for the larger biology community. Despite these laudable aims, providing adequate mentorship, training, opportunity, and supervision for undergraduate researchers is extraordinarily time-consuming and competed for the time and energy that faculty (particularly those with high teaching loads) have available for data analysis and communication of results. Consequently, all core faculty on this project voiced some version of this goal conflict, although their specific frustrations, preferences, and recommendations varied. They shared an appreciation for the science manager and emphasized that this staff position significantly contributed to the organization and function of the project as a whole. All core faculty suggested that additional personnel—or additional faculty time allocated through teaching release-would help in navigating this dual aim/goal conflict and further enhance network success. Based on this network's accomplishments, other recommendations that can support institutions and faculty engaging in networked research projects with undergraduates include: 1) establishing rigorous protocols to ensure data and database quality, 2) protecting personnel time to coordinate network and scientific processes, and 3) selecting appropriate partners and establishing explicit expectations for specific collaborations.

One final recommendation is needed. Our longitudinal research raises the question of what contributions are most valued within academia. This network, like other networked research projects, will create a scientific database while training the next generation of scientists. Nevertheless, traditional academic rewards tied to tenure and promotion may discourage cross-institutional and organizational collaboration (Nielsen, 2011; Binz-Scharf et al., 2014). Further, although the importance of incorporating undergraduate students into the research fold is lauded across scientific literature (Russell et al., 2007), and particularly for underrepresented students (Carpi et al., 2017), this work continues to be undervalued (Jones et al., 2018). We, along with other scholars (e.g., Mo et al., 2015) argue that academia must find a way to more effectively recognize and reward the scientific and pedagogical work that faculty contribute to undergraduate-focused networked research. This is a critical challenge as innovative networked research expands and new generations of scientists contribute to addressing humanity's grand challenges in increasingly transdisciplinary contexts.

\section{ACKNOWLEDGMENTS}

Support for this work was provided by a grant from the National Science Foundation (NSF IOS-1052262, IOS-1354771, and IOS-1355106). Its contents are solely the responsibility of the authors and do not necessarily represent the official views of the NSF. This study was reviewed and determined exempt by the UGA Institutional Review Board (no. STUDY00004081). We thank the members of the BRN (faculty, manager, and students) at the heart of this study. We thank Erin Dolan, Evan Conaway, Zoheb Sulaiman, and Jack Cherry for their contributions over 
the course of the project and insights toward our interpretation of data.

\section{REFERENCES}

Alkaher, I., \& Dolan, E. (2014). Integrating research into undergraduate cours es: Current practices and future directions. In Sunal, D., Sunal, C., Zollman, D., Mason, C., \& Wright, E. (Eds.), Research in science education: Research based undergraduate science teaching (pp. 403-434). Charlotte, NC: Information Age.

American Association for the Advancement of Science. (2011). Vision and change in undergraduate biology education: A call to action. Washington, DC.

Ankeny, R., \& Leonelli, S. (2012). What is so special about model organisms? Studies in History and Philosophy of Science, Part A, 42(2), 313-323.

Ankeny, R. A. (2000). Model organisms as models: Understanding the "lingua franca" of the Human Genome Project. Philosophy of Science, 68, 251261.

Ankeny, R. A. (2001). The natural history of Caenorhabditis elegans research. Nature Reviews Genetics, 2(6), 474-479.

Bangera, G., \& Brownell, S. E. (2014). Course-based undergraduate research experiences can make scientific research more inclusive. CBE-Life Sciences Education, 13(4), 602-606.

Barnosky, A. D., Ehrlich, P. R., \& Hadly, E. A. (2016). Avoiding collapse: Grand challenges for science and society to solve by 2050. Elementa: Science of the Anthropocene, 4, 94.

Binz-Scharf, M., Kalish, Y., \& Paik, L. (2014). Making science: New generations of collaborative knowledge production. American Behavioral Scientist, 59, 531-547.

Bos, N., Gergle, D., Olson, J., \& Olson, G. (2001). Being there versus seeing there: Trust via video. Paper presented at the CHI 2001, Seattle, WA, 291292.

Bos, N., Zimmerman, A., Olson, J., Yew, J., Yerkie, J., Dahl, E., \& Olson, G. (2008). From shared databases to communities of practice: A taxonomy of collaboratories. Journal of Computer-Mediated Communication, 12, $318-338$.

Boudreaux, M. J., \& Ozer, D. J. (2013). Goal conflict, goal striving, and psychological well-being. Motivation and Emotion, 37(3), 433-443.

Bowker, G. C., \& Star, S. L. (1999). Sorting things out: Classification and its consequences, Cambridge, MA: MIT Press.

Bowne, D., Downing, A., Hoopes, M., LoGiudice, K., Thomas, C., Anderson, L., ... \& Shea, K. (2011). Transforming ecological science at primarily undergraduate institutions through collaborative networks. BioScience, 61(5), 386-392.

Brownell, S., Kloser, M., Fukami, T., \& Shavelson, R. (2012). Undergraduate biology lab courses: Comparing the impact of traditionally based "cookbook" and authentic research-based courses on student lab experiences. Journal of College Science Teaching, 41, 18-27.

Carpi, A., Ronan, D., Falconer, H., \& Lents, N. (2017). Cultivating minority students: Undergraduate research increases self-efficacy and career ambitions for underrepresented students in STEM. Journal of Research in Science Teaching, 54, 169-194

Cosier, R. A., \& Rose, G. L. (1977). Cognitive conflict and goal conflict effects on task performance. Organizational Behavior and Human Performance 19(2), 378-391.

Creswell, J. W., \& Miller, D. L. (2000). Determining validity in qualitative inquiry. Theory into Practice, 39(3), 124-130.

Cummings, J., \& Kiesler, S. (2005). Collaborative research across disciplinary and organizational boundaries. Social Studies of Science, 35, 703-722.

Delamont, S., \& Atkinson, P. (2001). Doctoring uncertainty: Mastering craft knowledge. Social Studies of Science, 31, 87-107.

De Sanctis, G., \& Mong, P. R. (1998). Communication processes for virtual organizations. Journal of Computer-Mediated Communication, 3. https://onlinelibrary.wiley.com/doi/full/10.1111/j.1083-6101.1998. tb00083.x

de Solla Price, D. J. (1963). Little science, big science. New York: Columbia University Press.
Dolan, E., \& Johnson, D. (2010). The undergraduate-postgraduate-faculty triad: Unique functions and tensions associated with undergraduate research experiences at research universities. CBE-Life Sciences Education, 9, 543-553.

Flick, U. (2004). Triangulation in qualitative research. In Flick, U., von Kardoff, E., \& Steinke, I. (Eds.), A companion to qualitative research (pp. 178-183). Thousand Oaks, CA: Sage.

Greenwald, A. G., \& Banaji, M. R. (1995). Implicit social cognition: Attitudes, self-esteem, and stereotypes. Psychological Review, 102(1), 4-27.

Hanauer, D. I., \& Dolan, E. L. (2014). The Project Ownership Survey: Measuring differences in scientific inquiry experiences. CBE-Life Sciences Education, 13, 149-158

Hanauer, D. I., Graham, M., SEA-PHAGES, Betancur, L., Bobrownicki, A., Cresawn, S., ... \& Hatfull, G. (2017). An inclusive research education community (iREC). Proceedings of the National Academy of Sciences USA, 114, 13531-13536.

Hara, N., Solomon, P., Kim, S. L., \& Sonnenwald, D. H. (2003). An emerging view of scientific collaboration: Scientists' perspectives on collaboration and factors that impact collaboration. Journal of the American Society for Information Science and Technology, 54, 952-965.

Hey, T., \& Trefethen, A. (2008). E-science, cyber-infrastructure, and scholarly communication. In Olson, G., Zimmerman, A., \& Bos, N. (Eds.), Scientific collaboration on the Internet (pp. 15-33). Cambridge, MA: MIT Press.

Hinds, P. J., \& Kiesler, S. (2002). Distributed work. Cambridge, MA: MIT Press.

Jahn, T., Bergmann, M., \& Keil, F. (2012). Transdisciplinarity: Between mainstreaming and marginalization. Ecological Economics, 79, 1-10.

Jarvenpaa, S., \& Leidner, D. (1999). Communication and trust in global virtual teams. Organization Science, 10, 791-815.

Jones, K., Ankeny, R., \& Cook-Deegan, R. (2018). The Bermuda Triangle: The pragmatics, policies, and principles for data sharing in the history of the Human Genome Project. Journal of the History of Biology, 51, 693-805.

Kelly, R. E., Mansell, W., \& Wood, A. (2015). Goal conflict and well-being: A review and hierarchical model of goal conflict, ambivalence, self-discrepancy and self-concordance. Personality and Individual Differences, $85,212-229$

Latour, B., \& Woolgar, S. (1987). Laboratory life. Princeton, NJ: Princeton University Press.

Laursen, S., Hunter, A-B., Seymour, E., Thiry, H., \& Melton, G. (2010). Undergraduate research in the sciences: Engaging students in real science. San Francisco, CA: Jossey-Bass.

Leonelli, S. (2011). Packaging small facts for re-use: Databases in model organism biology. In Howlett, P., \& Morgan, S. (Eds.), How well do facts travel? The dissemination of reliable knowledge (pp. 325-248). Cambridge, MA: Cambridge University Press

Mo, G. Y., Hayat, Z., \& Wellman, B. (2015). How far can scholarly networks go? Examining the relationships between distance, disciplines, motivations, and clusters. Communication and Information Technologies Annual, 9 107-133.

Murren, C. J., Wolyniak, M. J., Rutter, M. T., Bisner, A. M., Callahan, H. S., Strand, A. E., \& Corwin, L. A. (2019). Undergraduates phenotyping Arabidopsis knockouts in a course-based undergraduate research experience: Exploring plant fitness and vigor using quantitative phenotyping methods. Journal of Microbiology \& Biology Education, 20, 1-10.

Nelson, N. (2017). Model behavior: Animal experiments, complexity, and the genetics of psychiatric disorders. Chicago: University of Chicago Press.

Nielsen, M. (2011). Reinventing discovery: The new era of networked science. Princeton, NJ: Princeton University Press.

Olson, G., \& Olson, J. (2003). Mitigating the effects of distance on collaborative intellectual work. Economic Innovation and New Technologies, 12 27-42.

Patton, M. Q. (2015). Qualitative research and methods: Integrating theory and practice. Thousand Oaks, CA: Sage.

Rainie, L., \& Wellman, B. (2012). Networked: The new social operating sys tem. Cambridge, MA: MIT Press. 
Russell, S., Hancock, M., \& McCullough, J. (2007). Benefits of undergraduate research experiences. Science, 316, 548-549.

Rutter, M. T., Murren, C. J., Callahan, H. S., Bisner, A. M., Leebens-Mack, J., Wolyniak, M. J., \& Strand, A. E. (2019). Distributed phenomics with the unPAK project reveals the effects of mutations. Plant Journal, 100, 199-211.

Saldaña, J. (2016). The coding manual for qualitative researchers. Thousand Oaks, CA: Sage.

Sarma, G. P., Lee, C., Portegys, T., Ghayoomie, V., Jacobs, J., Alicea, B., ... \& Larson, S. (2018). OpenWorm: Overview and recent advances in integrative biological simulation of Caenorhabditis elegans. Philosophical Transactions, 373, 20170382.

Simmons, J., Anderson, L., Bowne, D., Dosch, J., Gartner, T., Hoopes, M., ... \& Shea, K. (2016). Collaborative research networks provide unique opportunities for faculty and student researchers. Council on Undergraduate Research, 36(4), 12-18.
Sonnenwald, D. (2008). Scientific collaboration. Annual Review of Information Science and Technology, 41, 643-681.

Stein, L. (2001). Genome annotation: From sequence to biology. Nature Reviews Genetic, 2, 493-503.

Thompson, J. J., Conaway, E., \& Dolan, E. L. (2016). Undergraduate students' development of social, cultural, and human capital in a networked research experience. Cultural Studies of Science Education, 11, 959-990.

Thompson, J. J., \& Jensen-Ryan, D. (2018). Becoming a "science person": Faculty recognition and the development of cultural capital in the context of undergraduate biology research. CBE-Life Sciences Education, 17, 1-17.

Waring, T., Teisl, M., Manandhar, E., \& Anderson, M. (2014). On the travel emissions of sustainability science research. Sustainability, 6, 27182735. 